\title{
Educação Física na Educação Infantil: uma revisão sistemática
}

\section{Physical Education in Early Childhood Education: a systematic review}

\author{
Ivan Stein', Adriana Coutinho de Azevedo Guimarães'1, Allana Alexandre Cardoso', Zenite Machado' \\ 'Universidade do Estado de Santa Catarina (CEFID/UDESC), Florianópolis, SC, Brasil.
}

Recebido em: Dezembro 2015 / Aceito em: Dezembro 2015

allana.alexandre@gmail.com

\section{RESUMO}

A infância consiste em uma das etapas mais importantes da vida, pois a correta estimulação nos domínios motor, cognitivo e sócio afetivo nesta fase, acompanhará o indivíduo até a fase adulta. Objetivo: investigar quais aspectos da Educação Física (EF) na Educação Infantil são abordados nas publicações disponíveis na última década. Resultados: foram encontrados 232 artigos, sendo que os cinco avaliados na íntegra abordaram predominantemente o nível de atividade física e as recomendações a respeito das aulas de EF. Considerações finais: os estudos demonstraram que a EF contribui para o aumento do nível de atividade física e melhora aspectos do desenvolvimento motor na Educação Infantil.

Palavras-chave: Desenvolvimento Infantil; Educação Física; Educação Infantil.

\section{ABSTRACT}

Childhood is one of the most important stages of life, therefore the correct stimulation in the motor domain, cognitive and socio-emotional at this stage, follow the individual into adulthood. Objective: to investigate which aspects of physical education (PE) in Early Childhood Education are addressed in publications available in the last decade. Results: 232 articles were found, of which five fully evaluated predominantly approached the level of physical activity and recommendations regarding the PE classes. Closing remarks: the $P E$ showed that contributes to the increased level of physical activity and improves aspects of motor development in early childhood education.

Key words: Child Development; Physical Education; Early Childhood Education.

\section{INTRODUCÃO}

Os primeiros anos de vida constituem uma etapa importante do desenvolvimento humano, pois neste período ocorrem progressos em todos os domínios do desenvolvimento em um ritmo muito superior ao de qualquer outra fase da vida. Particularmente, até os cinco anos de idade, as crianças rapidamente desenvolvem capacidades fundamentais, as quais servirão de base para seu desenvolvimento posterior. São notáveis os avanços linguísticos e cognitivos, além do dramático progresso emocional, social, regulatório e moral.

Para que a criança cresça e se desenvolva de maneira adequada e saudável, é de extrema importância a sua inserção em creches ou pré-escolas, a fim de contribuir positivamente para o seu pleno desenvolvimento. Conforme dispõe o artigo 29 da Lei de Diretrizes e Bases da Educação Nacional - LDB, ${ }^{1}$ esta etapa, que compreende a educação infantil. É a primeira da educação básica e tem como finalidade o desenvolvimento integral da criança de zero a seis anos de idade, em seus aspectos físico, psicológico, intelectual e social, complementando a ação da família e da comunidade. Apesar de não haver obrigatoriedade de matrícula, a inclusão é um direito da criança e deve ser respeitado e incentivado.

Consoante a isto, o $3^{\circ}$ parágrafo do artigo 26 da lei 9394/96 garante o ensino da Educação Física (EF) nas escolas, ${ }^{2}$ sendo esta uma disciplina que compreende o indivíduo em sua totalidade, podendo assim contribuir para o desenvolvimento integral da criança, ${ }^{3}$ em um momento privilegiado da sua educação. O caráter de especificidade da EF, que se manifesta prioritariamente por meio do movimento, contribui para o aumento do nível de atividade física, resultando em melhorias da saúde física ${ }^{4}$ e mental. ${ }^{5}$

A importância do desenvolvimento motor ideal não deve ser minimizada ou considerada como secun- 
dária em relação a outros campos do desenvolvimento. ${ }^{6}$ Ressaltando ainda que o desenvolvimento motor representa um aspecto do processo de desenvolvimento integral e está intrinsecamente inter-relacionado às áreas cognitivas e afetivas do comportamento humano. ${ }^{6}$ Os benefícios advindos da atividade física em crianças com idade escolar sobre a cognição estão documentados de acordo com alguns autores. ${ }^{7}$ Isto acontece, pois no processo de desenvolvimento infantil e as mudanças ocorridas no sistema motor são acompanhadas por desenvolvimento do sistema cognitivo. Desta forma, é impossível dissociar esses dois aspectos do desenvolvimento, uma vez que ambos coexistem no mesmo organismo. ${ }^{8}$ No domínio sócio-afetivo, muitos fatores como emoções, sentimentos, cooperação, solidariedade, motivação entre outros, estão envolvidos.

Ao longo da primeira década de vida, as crianças têm potencial para realizar as habilidades motoras fundamentais com desenvoltura e adaptável ao contexto. ${ }^{9}$ Porém, para que tais potencialidades se concretizem, estas crianças precisam de estímulos ambientais na forma de instrução e prática apropriadas para que esse potencial seja plenamente revertido em ações motoras mais refinadas..$^{10}$ Estes estímulos ambientais podem ser observados em programas de atividade física e aulas de EF. ${ }^{11}$

Desta forma, a presente revisão baseou-se na necessidade da EF na Educação Infantil e sua importância para o desenvolvimento integral da criança, tendo como objetivo conhecer o que está sendo produzido sobre esta área do conhecimento, bem como investigar quais os aspectos desta área, na Educação Infantil, são abordados nas publicações disponíveis em bases de dados e bibliotecas digitais, na última década.

\section{MÉTODO}

O presente estudo caracteriza-se como uma revisão sistemática de trabalhos científicos que estudaram a EF na Educação Infantil e o seu momento na rotina da instituição escolar. Por tratar-se de uma pesquisa bibliográfica, com uso exclusivo de bases de dados públicos, esta dispensa a submissão ao Comitê de Ética em Pesquisa em Seres Humanos.

Nos critérios de elegibilidade foram incluídos estudos publicados nos últimos dez anos, com texto completo disponível; com restrição de idioma em português e inglês. Os artigos que avaliaram outras populações; não investigaram a EF na educação infantil; estudos em formato de Monografias, Teses, Dissertações, Revisões Sistemáticas e os artigos em duplicata foram excluídos.

Esta revisão foi realizada no período entre setembro e dezembro de 2014. Os estudos foram buscados nas bases de dados Sciverse Scopus, MEDLINE/ Pubmed (Medical Literature Analysis and Retrieval System on-line), LILACS (Literatura Latino-Americana e do Caribe em Ciências da Saúde), e na biblioteca digital SciELO (Scientific Electronic Library Online). Os descritores utilizados para a busca, conforme os descritores em ciências da saúde (DESCs) foram: “Educação Física e Educação Infantil" e "Physical education and preschool children". O procedimento de avaliação metodológica envolveu uma etapa prévia para análise dos dados e critérios de inclusão e exclusão, sendo que, para essa etapa, os artigos foram analisados de acordo com título e resumo. Posteriormente, os artigos avaliados na primeira etapa e que se apresentavam na íntegra foram lidos para que se coletassem as informações referentes à temática. Por fim, os estudos selecionados foram catalogados considerando-se autores, ano de publicação, língua original, periódicos, Qualis, bases, amostras, título do estudo, tipo de estudo, objetivos, instrumentos e principais resultados.

O processo de busca nas bases de dados eletrônicas e nas bibliotecas virtuais, seleção dos estudos, leitura dos artigos e compilação das informações foi realizado pelo pesquisador principal deste. Um segundo pesquisador replicou esse processo de busca e seleção dos artigos de forma cega e independente. Quando houve controvérsias, um terceiro pesquisador foi chamado para fazer a leitura e auxiliar neste processo.

A figura 1 apresenta o fluxograma da estratégia utilizada para a seleção de inclusão e exclusão dos estudos desta revisão sistemática, bem como os filtros utilizados para estes resultados.

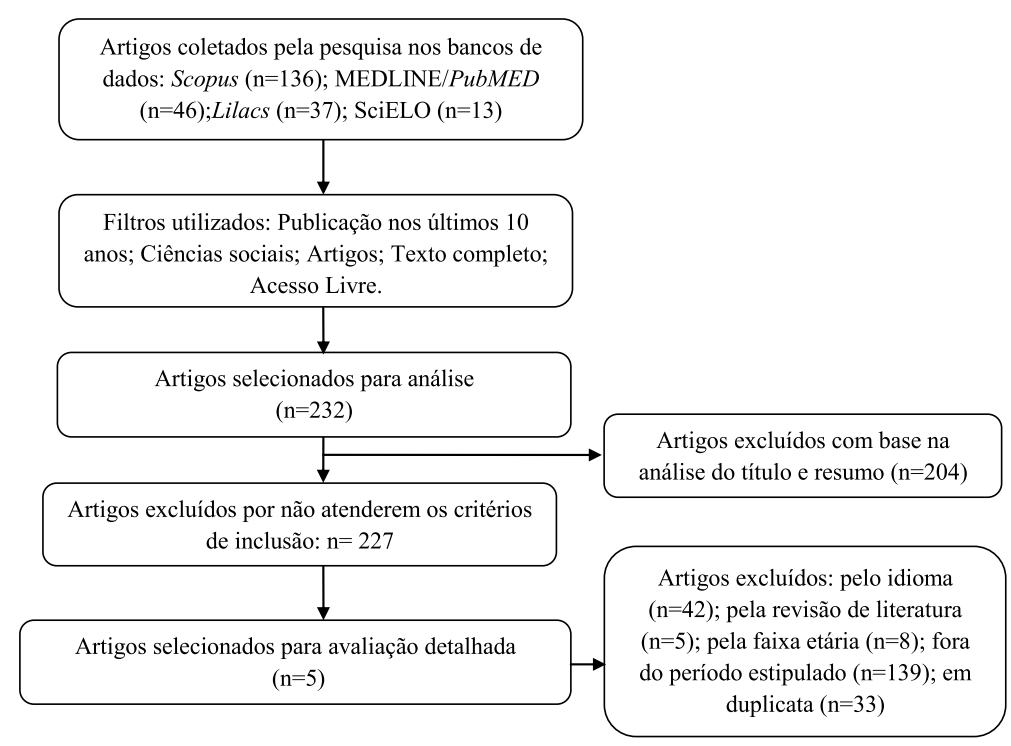

Figura 1 - Fluxograma dos estudos incluídos na revisão. 


\section{RESULTADOS}

A primeira etapa do estudo envolveu uma busca com a utilização dos termos “Educação Física e Educação Infantil" e "Physical education and preschool children", na qual foram verificadas as obras com livre acesso, na área das ciências sociais e que estavam disponíveis na íntegra e no formato de artigo. Dessa etapa resultou a seleção de 232 artigos. Em um segundo levantamento, foram identificados aqueles que apresentavam os termos de busca no título ou no resumo, resultando em 204 publicações rejeitadas. Outras exclusões ocorreram em função da inadequação do idioma ( $n=42)$, tratar-se de estudos de revisão $(n=5)$, extrapolação da faixa etária da Educação Infantil ( $=8$ ), data de publicação anterior ao limite estabelecido ( $n=139$ ) e com publicação foi encontrada duplicada ( $n=33$ ).

Desta forma, apenas cinco publicações atenderam aos critérios de inclusão desta revisão sistemática, incidindo em dois artigos na base LILACS, dois na base PubMED, um na base SciELO, sendo este encontrado em duplicata na base LILACS, não podendo ser incluído na amostra, e um na base SCOPUS, os quais foram lidos na íntegra, pois apresentaram o texto completo disponível online.

O Quadro 1 apresenta os estudos selecionados nesta revisão sistemática. Destes textos selecionados, todos foram publicados a partir de 2010, indicando um aumento na produção referente a esta área nos últimos cinco anos, observando-se o período estipulado para as publicações incluídas nesta pesquisa, de 2004 a 2014.

Em relação ao idioma, nota-se um desequilíbrio entre a língua inglesa e a portuguesa, com grande vantagem para os textos em inglês, quatro foram publicados em inglês, e apenas um em português. Esses resultados sugerem que o interesse dos pesquisadores a respeito da temática EF e Educação Infantil, que publicaram na língua materna, é reduzido.

Nos periódicos, no que se refere à classificação dos artigos (Qualis - Capes), houve bastante diversidade, tendo sido encontrado apenas um artigo para as seguintes designações: A1, A2, B1, B3 e C.

As bases de dados, LILACS e PubMed e SCOPUS são bases de dados em ciências da saúde, literatura biomédica e multidisciplinar, respectivamente. Entre os artigos selecionados, dois foram extraídos na base
LILACS, dois na PubMed, e apenas um artigo na base SCOPUS. Um dos artigos encontrados na base SciELO ocorreu como referência cruzada com a base LILACS, deixando esta base sem representação neste trabalho.

O Quadro 2 refere-se ao detalhamento dos estudos selecionados quanto aos objetivos, instrumentos utilizados e principais resultados. Observa-se grande variedade no tamanho amostral, abordando entre 71 até 1042 participantes, totalizando 2575 crianças, distribuídas homogeneamente entre meninos e meninas. Nos títulos destes cinco estudos, se percebe grande utilização do termo atividade física (estudos: 2, 3, 4 e $5)$, que representam $80 \%$ dos textos selecionados para este trabalho. Apenas um artigo (estudo: 1) apresentou o tema desenvolvimento motor.

Nos tipos de estudos, foi observado que três utilizaram métodos, envolvendo estudos transversais em suas pesquisas, respectivamente os estudos 2,4 e 5 . Os demais, estudos 1 e 3, envolveram programas de intervenções. O estudo 1 compreendeu um programa que consistiu em 32 sessões com duração de 45 minutos, durante oito semanas. O estudo 2, de corte transversal foi parte integrante do projeto ELOS-Pré (Estudo Longitudinal de Observação da Saúde e Bem-estar da Criança em Idade Pré-escolar). O estudo 3 utilizou um ensaio clinico randomizado por cluster, realizando um programa no qual se estabeleceu aulas diárias de EF com 30 minutos de duração, durante todo ano letivo. Estas crianças foram avaliadas antes das interferências e ao fim do programa. O estudo 4 observou as atividades realizadas em cinco dias consecutivos de aula (de segunda à sexta-feira) entre fevereiro e dezembro de 2008, durante o horário escolar para um mínimo de $6 \mathrm{~h}$ por dia. O estudo 5 acompanhou os pré-escolares durante as aulas de EF na pré-escola.

Os objetivos dos trabalhos selecionados apresentaram interesses diferenciados. O estudo 1 investigou os efeitos das intervenções por meio de jogo, com orientação e jogo livre em ambiente enriquecido sobre o desenvolvimento de habilidades motoras de crianças pré-escolares (este artigo tem preocupações voltadas para o desenvolvimento motor). O estudo 2 descreveu as características do ambiente das escolas de educação infantil e analisou a associação de fatores ambientais com o nível de atividade física (AF) de crianças pré-escolares. O estudo 3 promoveu o aumento do nível

Quadro 1 - Estudos que investigaram a Educação física na Educação infantil.

\begin{tabular}{|c|c|c|c|c|c|}
\hline Estudos/Autor/Ano & Língua Original & Periódico/Qualis & Tipo de estudo & Amostra (n) & Média de idade em anos \\
\hline $\begin{array}{l}\text { Palma, Pereira, } \\
\text { Valentininit }^{14}\end{array}$ & Português & $\begin{array}{l}\text { Motriz Revista de Educação } \\
\text { Física. UNESP (A2) }\end{array}$ & $\begin{array}{l}\text { Transversal com } \\
\text { intervenção }\end{array}$ & $\begin{array}{l}71 \text { crianças, distribuí- } \\
\text { das em GC e grupo } \\
\text { experimental }\end{array}$ & $\mathrm{NI}^{*}$ \\
\hline $\begin{array}{l}\text { Roth, Mauer, Obinger, } \\
\text { Ruf, Graf, Kriemler, Lenz, } \\
\text { Lehmacher, Hebestreit }{ }^{16}\end{array}$ & Inglês & Public Health (A1) & $\begin{array}{l}\text { Ensaio clínico } \\
\text { randomizado por } \\
\text { cluster }\end{array}$ & $\begin{array}{l}696 \text { crianças } 348 \text { no } \\
\text { grupo de intervenção } \\
\text { e } 348 \text { no GC }\end{array}$ & 5 a 6 \\
\hline $\begin{array}{l}\text { Van Cauwenberghe, Gub- } \\
\text { bels, Labarque, De Bour- } \\
\text { deaudhuij, Cardon }\end{array}$ & Inglês & $\begin{array}{l}\text { Early Childhood Research } \\
\text { Quarterly (C) }\end{array}$ & Transversal & $\begin{array}{l}573 \text { pré-escolares } \\
\text { (288 meninos) }\end{array}$ & 5 a 4 \\
\hline
\end{tabular}

*NI: Não informado 


\begin{tabular}{|c|c|c|}
\hline Estudo/objetivos & Instrumento & Resultados \\
\hline $\begin{array}{l}\text { Investigar os efeitos das inter- } \\
\text { venções jogo com orientação e } \\
\text { jogo livre no desenvolvimento } \\
\text { de habilidades motoras em } \\
\text { pré-escolares. }\end{array}$ & $\begin{array}{l}\text { Test of Gross Motor Development, antes } \\
\text { e após o período interventivo. }\end{array}$ & $\begin{array}{l}\text { Os meninos e meninas do grupo jogam com orientação apresentaram ganhos em } \\
\text { habilidades motoras; Mudanças não foram observadas nos meninos e meninas que } \\
\text { constituíram o grupo jogo livre em ambiente enriquecido, nem nos do grupo controle; } \\
\text { O papel do professor no grupo "jogo com orientação" foi determinante para a melhora } \\
\text { de desempenho das crianças pré-escolares. }\end{array}$ \\
\hline $\begin{array}{l}\text { Descrever características do } \\
\text { ambiente das escolas de edu- } \\
\text { cação infantil e analisar a as- } \\
\text { sociação de fatores ambientais } \\
\text { com o nível de AF de crianças } \\
\text { pré-escolares. }\end{array}$ & $\begin{array}{l}\text { Entrevista com os pais. A avaliação do } \\
\text { ambiente da escola foi efetuada por meio } \\
\text { de um inventário. }\end{array}$ & $\begin{array}{l}\text { Nas escolas de menor porte ( } \leq 100 \text { crianças) nenhum dos fatores ambientais avali- } \\
\text { ados apresentou associação com o nível de AF em dias de semana. Nas escolas de } \\
\text { maior porte ( }>100 \text { crianças), } 5 \text { características ambientais foram identificadas como } \\
\text { fatores inversamente associados ao nível de AF: oferta de aula de EF; realização de } \\
\text { pelo menos } 1 \text { recreio por dia; AF orientada durante o recreio; permissão para que as } \\
\text { crianças tragam brinquedos no recreio; oferecimento de AF supervisionada. As carac- } \\
\text { terísticas ambientais estão associadas à pratica de AF em pré-escolares, mas somente } \\
\text { a oferta de recreios foi tida como fator de proteção para baixo nível de AF. }\end{array}$ \\
\hline $\begin{array}{l}\text { Investigar a eficácia e a viabi- } \\
\text { lidade de uma intervenção por } \\
\text { meio de AF para promover a } \\
\text { AF, determinados critérios de } \\
\text { saúde e utilização de mídias } \\
\text { em crianças. }\end{array}$ & $\begin{array}{l}\text { Massa corporal, estatura e dobras } \\
\text { cutâneas; Método oscilométrico e auscul- } \\
\text { tatório; Plataforma de força; KMS } 3-6^{*} \text {; } \\
\text { MOT } 4-6^{*} \text {. }\end{array}$ & $\begin{array}{l}\text { Aumento no percentual de tempo gasto em AF moderada e vigorosa; Melhoria } \\
\text { no escores em testes de habilidades motoras (transposição de obstáculos, } \\
\text { equilíbrio estático em plataforma de força, equilíbrio monopedal, salto horizontal, } \\
\text { salto lateral); Melhoria na flexibilidade; Redução do IMC, dobras cutâneas, pressão } \\
\text { arterial; Redução na frequência de acidentes e infecções; Redução na utilização } \\
\text { de mídias eletrônicas; Em função dos resultados o programa de intervenção será } \\
\text { implementado em toda a Alemanha. }\end{array}$ \\
\hline $\begin{array}{l}\text { Analisar diferenças entre AF } \\
\text { total e AF de moderada a } \\
\text { vigorosa em pré-escolares em } \\
\text { dias com e sem aulas de EF e } \\
\text { acessar a contribuição dessas } \\
\text { aulas no total de AF no período } \\
\text { escolar. }\end{array}$ & $\begin{array}{l}\text { Massa corporal e estatura;Acelerômetro } \\
\text { durante } 5 \text { dias de aula consecutivos; } \\
\text { System for Observing Fitness Instruction } \\
\text { Time (SOFIT) }\end{array}$ & $\begin{array}{l}\text { A aula de EF contribui com a média de } 27,7 \% \text { para o total de AF e } 32.8 \% \text { para AF de } \\
\text { moderada a vigorosa durante os dias com aula de EF; Durante um dia com aula de EF } \\
\text { as crianças se envolvem significativamente mais e em AF total e MVPA ( } 66.40 \text { min; } \\
26.55 \text { min), quando se compara com dias sem aula de EF ( } 55.45 \text { min; } 20.16 \text { min) (P } \\
<0.05 \text { ). Isso representa um aumento de TPA e MVPA em } 19.5 \% \text { e } 31.8 \% \text { entre as } \\
\text { meninas e } 19.2 \% \text { e } 33.4 \% \text { entre os garotos, respectivamente, durante a aula de EF. }\end{array}$ \\
\hline $\begin{array}{l}\text { Examinar o nível de AF de } \\
\text { pré-escolares e a associação } \\
\text { com o contexto da aula, o } \\
\text { comportamento do professor e } \\
\text { fatores ambientais durante as } \\
\text { aulas de EF. }\end{array}$ & $\begin{array}{l}\text { Gravação das sessões em vídeo; acel- } \\
\text { erômetro; entrevista; trena; consulta ao } \\
\text { banco de dados pessoais dos escolares. }\end{array}$ & $\begin{array}{l}\text { Pré-escolares acumularam } 12 \text { min (33\%) de moderada a vigorosa AF, (MVPA), } 5 \text { min } \\
\text { (13\%) leve AF, e } 20 \text { min (54\%) de comportamento sedentário na aula de EF. } 47 \% \text { da } \\
\text { variância da AF (MVPA) podem ser atribuídas a diferenças nas aulas de EF: menor con- } \\
\text { hecimento de conteúdo, menor incentivo, menor gerenciamento, menor densidade de } \\
\text { crianças por m2, utilização de materiais/equipamentos de obstrução e uso de equipa- } \\
\text { mentos foram significativamente associados com maior MVPA níveis. O formato atual } \\
\text { das aulas de EF contribui apenas com uma pequena parcela para o nível adequado de } \\
\text { AF recomendado para pré-escolares. }\end{array}$ \\
\hline
\end{tabular}

AF: atividade física; EF: educação física; MVPA: atividade física moderada a vigorosa; *KMS 3 - 6: Karlsruher Motorik-Screening für Kindergartenkinder; * MOT 4-6: Motoriktest für vier- bis sechsjährige Kinder

de AF e habilidades motoras em crianças participantes de um programa de AF escolar, reduzindo os fatores de risco à saúde, bem como o uso da mídia. No estudo 4 os autores objetivaram analisar diferenças entre AF total e AF de moderada a vigorosa em pré-escolares em dias com aula de EF e dias sem aula de EF e acessar a contribuição dessas aulas no total de AF, no período escolar. Finalmente, o estudo 5 teve como meta examinar o nível de AF de pré-escolares e a associação com o contexto da aula, o comportamento do professor e fatores ambientais, durante as aulas de EF.

Quanto aos instrumentos, o estudo 1 utilizou o Test of Gross Motor Development - 2 (TGMD-2), ${ }^{17}$ para avaliar os padrões motores locomotores dos participantes. O estudo 2 aplicou um questionário com os pais e/ ou responsáveis pelas crianças, na forma de entrevista face à face (entrevista com os pais). A avaliação do ambiente da escola foi efetuada por meio de um inventário desenvolvido pelos pesquisadores. O estudo 3 desenvolveu uma intervenção constituída de sessões diárias de Educação Física, com duração de 30 minutos, durante um ano letivo, ministrada por profissionais supervisionados pelos investigadores, utilizando acelerômetros; testes de habilidades motoras específicas (transposição de obstáculo, equilíbrio sobre um pé, equilíbrio à reta- guarda, saltos laterais, salto em distância), flexibilidade (sentar e alcançar); força de reação ao solo; índice de massa corporal (IMC); pressão arterial; batimentos cardíacos; levantamento da ocorrência de acidentes ou infecções. O estudo 4 também utilizou acelerômetros (em uma frequência de cinco dias consecutivos) e para além disso, fez uso de medidas antropométricas (massa corporal e estatura). Por fim, estudo 5 foi o terceiro estudo incluído nesta revisão sistemática que fez uso de acelerômetros para investigar sua amostra, utilizou também a gravação das sessões em vídeo, entrevistas semiestruturadas, trena para medição dos locais onde as atividades eram realizadas e consulta ao banco de dados pessoais dos escolares.

Dentre os principais resultados, destacamos o fato de todos os estudos se preocuparem em retratar a importância de aulas de EF bem estruturadas, planejadas, com maior frequência semanal e ministradas em ambientes favoráveis para sua realização. Corroborando a isto, o estudo 1 evidenciou que o papel do professor de $E F$, no grupo jogo orientado, foi determinante para a melhora de desempenho das crianças. O estudo 2 sugeriu que as características do ambiente das escolas estão associadas ao nível de AF em crianças pré-escolares e que este aspecto deve ser mais investigado para gerar 
suporte teórico para a realização de ações de saúde publica, visando a promoção de comportamentos fisicamente ativos na escola e fora dela, nessa população especifica. $O$ estudo 3 verificou melhoria em testes de habilidades motoras, flexibilidade, IMC, dobras cutâneas, pressão arterial, frequência de acidentes e infecções, redução na utilização de mídias eletrônicas em crianças de 5 a 6 anos. Cabe ressaltar que, em função destes resultados, o programa de intervenção utilizado neste estudo será implementado em toda a Alemanha. $\mathrm{O}$ estudo 4 obteve que as aulas de EF contribuem para um aumento dos níveis de AF, quando comparados aos dias em que não havia aula de EF na escola. Em contrapartida, o estudo 5 discutiu o formato atual das aulas de EF, alertando que em sua pesquisa, a contribuição desta disciplina foi apenas uma pequena parcela para o nível adequado de AF recomendado para pré-escolares.

\section{DISCUS5ÃO}

Este trabalho teve como objetivo investigar quais os aspectos da EF na Educação Infantil foram evidenciados nas publicações disponíveis em bases de dados e bibliotecas digitais na última década e de que forma são desenvolvidos neste contexto. Apesar do grande lapso sem produções entre 2004 e 2010, e também a fraca produção cientifica encontrada a respeito do tema EF, na Educação Infantil, evidenciando o pouco interesse dos pesquisadores nesta área.

Entretanto, nos poucos estudos encontrados sobre o tema, a partir da literatura analisada, foi possível observar que há uma maior incidência de estudos relacionados à atividade física (AF) e seus benefícios para a saúde infantil, reforçando o objetivo abordado por quatro, dos cinco artigos selecionados. Acredita-se que esses resultados possam estar relacionados às bases de dados elegidas para a busca, pois apenas a base SCOPUS é multidisciplinar, enquanto as demais são voltadas à área da saúde. Essa relação entre as aulas de EF e os níveis de AF indicam uma crescente preocupação dos pesquisadores, o que pode estar ligado ao aumento mundial nos casos de obesidade infantil ${ }^{18} \mathrm{e}$ suas comorbidades associadas, ${ }^{19}$ bem como a ligação destes aspectos ao sedentarismo infantil e ao aumento de problemas de saúde relacionados aos baixos índices de AF. ${ }^{20}$ Fatos também apontados nos estudos de Roth et al., ${ }^{14}$ Vale et al. ${ }^{15}$ e Cauwenberghe et al. ${ }^{16}$

As modificações ocorridas no estilo de vida em razão do avanço tecnológico, em prol da promoção de um maior conforto para o ser humano, evidenciam a substituição da atividade humana pelas facilidades mecânicas, elétricas e eletrônicas, com as suas funções desempenhadas sentadas à frente de suas mesas, máquinas e computadores, acarretando em limitações de movimentos ${ }^{21}$ e no desenvolvimento da morbidade associada à presença de obesidade, doenças não infecciosas e crônico-degenerativas. ${ }^{22}$ Apesar da relevância deste assunto, a prevalência de exposição a baixos níveis de AF é alta e torna-se cada vez mais preocupante a sua redução durante a infância e adolescência, uma vez sabido dos inúmeros benefícios da $A F$, principalmente, quando associados à saúde esquelética (conteúdo mineral e densidade óssea) e ao controle da pressão sanguínea e da obesidade ${ }^{23}$ nesta faixa etária.

Neste sentido, acredita-se que o ambiente escolar seja de fundamental importância e propício para adoção e manutenção de comportamentos saudáveis, principalmente durante as aulas de Educação Física, que proporciona ao aluno, a conscientização da importância de se adquirir hábitos saudáveis e o respeito ao próprio corpo..$^{13}$ São amplamente conhecidas as recomendações da Associação Americana de Cardiologia (American Heart Association - AHA), para que as escolas garantam a prática de AF, durante a permanência das crianças no ambiente escolar, inclusive em seus intervalos, que se busque uma melhor qualificação dos professores de EF, que sejam promovidos eventos interescolares e desenvolvam programas de AF antes e depois dos horários de aulas. ${ }^{24}$

Mélo et al., ${ }^{13}$ Vale et al..$^{15}$ e Cauwenberghe et al. ${ }^{16}$ discutem em seus estudos sobre a pratica de AF e sua frequência de acordo com as necessidades de crianças e jovens está em torno de 60 minutos diários e com frequência de no mínimo três vezes por semana. ${ }^{25}$ Estas atividades devem ser apropriadas ao desenvolvimento de cada idade, agradáveis, divertidas e diversificadas em exercícios aeróbios, resistidos, de flexibilidade, coordenação, agilidade e equilíbrio. ${ }^{26}$

Outro aspecto a ser discutido aponta para a importância de planejar o currículo de EF para a Educação Infantil, visando o envolvimento das crianças em agradáveis e valiosas atividades e experiências observadas em Palma et al. ${ }^{12} \mathrm{Em}$ seu trabalho, os autores evidenciam o papel preponderante do professor de EF, demonstrando que os alunos que participaram de aulas orientadas obtiveram desempenho superior referente aos que tiveram aulas livres, mesmo estando em um ambiente enriquecido. Resultados semelhantes foram encontrados em Cotrim et al., ${ }^{11}$ Goodway e Branta, ${ }^{27}$ e Palma et al. ${ }^{28}$

A contribuição das aulas de EF estruturadas e planejadas também foi observada nos outros estudos selecionados, ${ }^{13-16}$ apontando a necessidade de alterações no ambiente, duração e na frequência semanal das aulas. Por conseguinte, essas mudanças favorecem a aquisição de habilidades motoras e o aumento da AF, sendo de grande importância para a prevenção relacionada à saúde e também para desenvolvimento normal na infância. A promoção de atividade física e habilidades motoras ganham cada vez mais importância na Educação Infantil, na atualidade. ${ }^{29} \mathrm{~A}$ inatividade física das crianças foi classificada como um fator modificável de risco para doenças relacionadas com o estilo de vida, e muitos dos fatores de risco conhecidos para doenças crônicas também são apresentados nestes estudos. Além disso, tem sido sugerido que a inatividade física, durante a juventude, está ligada a vários riscos relacionados para a saúde na vida adulta. ${ }^{15}$ Resultados destes estudos, ${ }^{13-16}$ sugerem que o AF estruturado, durante as aulas de EF, podem aumentar os níveis diários AF em pré-escolares, enfatizando a importância de aulas de educação física nestas idades.

Entretanto, as normativas a respeito da EF, na Educação Infantil, não apresentam uma regulamentação clara. Os profissionais desta área não contam com suficiente proteção legal para realizar suas atividades, sendo pouco reconhecidos socialmente, havendo também especialistas 
de outras áreas que realizam intervenções que seriam da competência exclusiva dos profissionais de EF. ${ }^{30}$

Outra problemática evidenciada para além da falta de produção cientifica acerca do tema, é o fato de que os pesquisadores ainda não conseguiram superar o hiato entre suas elaborações acadêmicas e as condições enfrentadas pelos profissionais de EF atuantes no ambiente escolar. Torna-se necessário, portanto, fazer chegar ao conhecimento e ao domínio destes professores os benefícios daquilo que tem sido produzido no âmbito acadêmico.

\section{CONSIDERACִÕES FINAIS}

Os estudos corroboram em seus resultados na necessidade das aulas de EF, na Educação Infantil. Todavia, preconizam que essas aulas sejam melhor estruturadas em um planejamento curricular adequado, com mais tempo e frequência durante a semana, e que ocorram em ambientes que favoreçam para a sua realização. Pode-se afirmar que os estudos acreditam na eficácia e importância das aulas de EF na Educação Infantil, fato evidenciado e reforçado nos principais resultados, no que se refere ao aumento do nível de atividade física, desenvolvimento de habilidades motoras e a manutenção da saúde destas crianças por meio das aulas de EF.

De forma geral, percebeu-se uma lacuna de conhecimento a respeito do tema, mesmo considerando o pequeno aumento de publicações a partir de 2010, demonstrando que esse ainda apresenta-se insuficiente à produção científica abordando a temática EF na Educação Infantil. Deste modo, a realização de futuras investigações é requerida, no intuito de confirmar estes achados e impactar no aumento da produção cientifica e transferência destes resultados para o ambiente escolar, consequentemente contribuindo e colaborando para uma valorização e reconhecimento da área e dos professores de EF.

\section{REFERÊNCIAS}

1. Brasil. Lei Darcy Ribeiro (1996)]. LDB: Lei de Diretrizes e Bases da Educação Nacional: lei no 9.394, de 20 de dezembro de 1996, que estabelece as diretrizes e bases da educação nacional. 2010; 5. ed. - Brasília: Câmara dos Deputados, Coordenação Edições Câmara, 2010.

2. Brasil. Senado Federal. Lei de Diretrizes e Bases da Educação Nacional: nº 9394/96. Brasília: 1996.

3. Sallis JF, Mckenzie TL. Physical education's role in public health. Research Quarterly for Exercise and Sport 1991;62(2):124-137.

4. Andersen LB, Riddoch C, Kriemler S, Hills A. Physical activity and cardiovascular risk factors in children. British Journal of Sports Medicine 2001;45:871-876. DOI: 10.1136/ bjsports-2011-090333.

5. Biddle SJH, Asare M. Physical activity and mental health in children and adolescents: a review of reviews. British Journal of Sports Medicine 2011;45:886-895. DOI: 10.1136/ bjsports-2011-090185.

6. Gallahue D, Ozmun JC. Compreendendo o Desenvolvimento Motor: bebês, crianças, adolescentes e adultos. São Paulo: Phorte, 2002.
7. Hillman $\mathrm{CH}$, Castellii DM, Buck $\mathrm{S} M$. Aerobic fitness and neurocognitive function in healthy preadolescent children. Medicine and Science in Sports and Exercise 2005;37:19671974. DOI: 0.1249/01.mss.0000176680.79702.ce.

8. Cardeal CM, Pereira LA, Silva PF, França NM. Efeito de um programa escolar de estimulação motora sobre desempenho da função executiva e atenção em crianças. Revista Motricidade 2013;9(3):44-56. DOI: http://dx.doi. org/10.6063/motricidade.9(3).762.

9. Gallahue DL. Conceitos para maximizar o desenvolvimento da habilidade de movimento especializado. Revista de Educação Física/UEM 2005;16(2):197-202. DOI: http:// dx.doi.org/10.4025/reveducfisv16n2p.

10. Rodrigues D, Avigo EL, Leite MMV, Bussolin RA, Barela JA. Desenvolvimento motor e crescimento somático de crianças com diferentes contextos no ensino infantile. Motriz, Rio Claro 2001:19(3):S49-S56.

11. Cotrim JR, Lemos AG, Néri-JR JE, Barela, J. A. Desenvolvimento de habilidades motoras fundamentais em crianças com diferentes contextos escolares. Revista de Educação Física/UEM 2011;22(4):523-533. DOI: 10.4025/ reveducfis.v22i4.12575.

12. Palma MS, Pereira BO, Valentini NC. Guided play and free play in an enriched environment: Impact on motor development. Revista Motriz, Rio Claro 2014;20:177-185. DOI: http:// dx.doi.org/10.1590/S1980-65742014000200007.

13. Mélo EM, Barros MVG, Hardman CM, Siqueira ML, Júnior RSW, Oliveira ESA. Associação entre o ambiente da escola de educação infantil e o nível de atividade física de crianças pré-escolares. Revista Brasileira de Atividade Física \& Saúde 2013;18(1):53-62.

14. Roth $\mathrm{K}$, Mauer $\mathrm{S}$, Obinger M, Ruf KC, Graf C, Kriemler S, Lenz $D$, Lehmacher $W$, Hebestreit $H$. Prevention through Activity in Kindergarten Trial (PAKT): A cluster randomised controlled trial to assess the effects of an activity intervention in preschool children. BioMed Central, Public Health 2010;10:410. DOI: 10.1186/1471-2458-10-410.

15. Vale S, Santos R, Miranda LS, Silva P, Mota J. The importance of physical education classes in pre-school children. Journal of Pediatrics and Child Health 2010;47:48-53. DOI: 10.1111/j.1440-1754.2010.01890.x.

16. Cauwenberghe EV, Labarque V, Gubbels J, Bourdeaudhuij ID, Cardon G. Preschooler's physical activity levels and associations with lesson context, teacher's behavior, and environment during preschool physical education. Early Childhood Research Quarterly 2011;27:221-230. DOI: 10.1016/j.ecresq.2011.09.007.

17. Ulrich D. The test of Gross motor development. 2 ed. Austin: Prod- Ed, 2000.

18. Pergher RNQ. et al. O diagnóstico de síndrome metabólica é aplicável às crianças? Jornal de Pediatria 2010;86(2):101108. DOI: http://dx.doi.org/10.2223/JPED.1983.

19. Pollock ML, Wilmore JH. Exercício na saúde e na doença: avaliação e prescrição para prevenção e reabilitação. Rio de Janeiro: MEDSI, 1993.

20. Ferreira MS, Najar AL. Programas e campanhas de promoção da atividade física. Ciência \& Saúde Coletiva 2005;10:207-19.

21. Rossetto AA. Utilização do centro desportivo da Universidade Estadual de Campinas: aspirações da comunidade. Dissertação de Mestrado. Piracicaba: UNIMEP, p. 66, 1990.

22. Romanella NE et al. Phisycal activity and attitudes in lean and obese children and their mothers. International Journal of Obesity 1991;15:407-414. DOI: 10.1186/1471-2458-13-895. 
23. Koezuka N, Koo M, Alisson KR, Adlaf EM, Dwyer JJM, Faulkner $\mathrm{G}$, et al. The relationship between sedentary activities and physical inactivity among adolescents: Results form the Canadian community health survey. Journal of Adolescent Health 2006;39(4):515-522. DOI: http://dx.doi org/10.1016/j.jadohealth.2006.02.005.

24. Pate RR, Davis MG, Robinson TN, Stone EJ, Mckenzie TL, Young JC. Promoting Physical Activity in Children and Youth: A Leadership Role for Schools: A Scientific Statement From the American Heart Association Council on Nutrition, Physical Activity, and Metabolism (Physical Activity Committee) in Collaboration With the Councils on Cardiovascular Disease in the Young and Cardiovascular Nursing. Circulation 2006; 114(11):1214-1224.

25. World Health Organization. Global Recommendations on Physical Activity for Health: WHO 2010.

26. Mendes R, Sousa N, Themudo Barata JL. Actividade Física e Saúde Pública: Recomendações para a Prescrição de Exercício.
Revista Acta Medica Portuguesa 2011;24(6):1025-1030.

27. Goodway J, Branta C. Influence of a motor skill intervention on fundamental motor skill development of disadvantaged preschool children. Research Quarterly for Exercise and Sport 2003;74(1):3646. DOI: 10.1080/02701367.2003.10609062.

28. Palma M, Camargo V, Pontes M. Efeitos da atividade física sistemática sobre o desenvolvimento motor de crianças préescolares. Revista da Educação Física/UEM 2012;23(3):421 429. DOI: http://dx.doi.org/10.4025/reveducfis.v23i3.14306.

29. Brasil. Ministério da Educação e do Desporto. Secretaria de Educação Fundamental. Referencial curricular nacional para a educação infantil /Ministério da Educação e do Desporto, Secretaria de Educação Fundamental. - Brasília: MEC/SEF, 1998.

30. Lovisolo H. Hegemonia e legitimidade nas ciências dos esportes. Motus Corporis: Revista de Divulgação Científica do Mestrado e Doutorado em Educação Física. Rio de Janeiro: Universidade Gama Filho 1996;3(2):52 